E1 contexto de la trampa: análisis experimental de los determinantes de la honestidad en Buriticá, Colombia

Adolfo Eslava y Estefanía García-Luna 
Lecturas de Economía, 92 (enero-junio 2020), pp. 9-32

Adolfo Eslava y Estefanía García-Luna

El contexto de la trampa: análisis experimental de los determinantes de la honestidad en Buriticá, Colombia

Resumen: La trampa es una acción recurrente en el comportamiento de los individuos y produce altos costos en la sociedad. Esto ha motivado su estudio reciente dentro de la economia y otras ciencias sociales, con el fin de encontrar los factores que inciden en ella y asi tratar de mitigar sus efectos. Con este objetivo, la presente investigación estudia la disposición a la trampa en el municipio de Buriticá (Antioquia, Colombia) a través de un experimento económico que confirma la presencia de acciones deshonestas en las decisiones de los participantes; además, se hace un análisis de los determinantes de la misma a través de un probit ordenado. El estudio revela que existe una relación entre las decisiones de los individuos y el contexto en el que se desenvuelven, por lo que factores como la percepción de riqueza, la confianza institucional y la victimización son determinantes. Esta investigación contribuye asi a los estudios previos sobre la toma de decisiones económicas, los cuales contradicen el modelo clásico de la maximización de beneficios.

Palabras clave: comportamiento; honestidad; trampa; experimentos.

Clasificación JEL: D01, D73, C93.

Context dependent cheating: experimental analysis of the determinants of honesty in Buriticá, Colombia

Abstract: Cheating is a regular action in people's behavior which produces high costs in society. For this reason, studies on cheating have been motivated in economics and other social sciences. In general, there is an aim to explain which factors are determinants of cheating in order to know how to mitigate its effects. In this context, this research studies the willingness to cheat in Buritica (Antioquia) through an economic field experiment that confirms the presence of dishonesty in the participant's decisions. In addition, an analysis of the determinants of cheating is made through an Ordered Probit where a causal relation is found between people's decisions and the context they live in -their perception of wealth, institutional trust and victimization - thus contributing to previous studies on economic decision making that contradict the classical model of maximizing benefits.

Keywords: behavior; honesty; cheating; experiments.

JEL Classification: D01, D73, C93.

Le contexte de la tricherie: analyse expérimentale des déterminants de l'honnêteté à Buriticá, Colombie

Résumé: La triche est une action récurrente dans le comportement des individus et elle génère des coûts élevés dans la société. Cela a motivé sa récente étude au sein de l'économie et d'autres sciences sociales, afin de trouver ses incitations et ainsi essayer d'atténuer ses effets. Dans cet objectif, la présente enquête étudie la disposition à tricher dans la municipalité de Buriticá (Antioquia, Colombie), à travers une expérimentation économique qui confirme la présence d'actions malhonnêtes dans les décisions des participants. En outre, une analyse des déterminants d'actions malhonnêtes est effectuée au moyen d'un modèle probit ordonné. L'étude révèle qu'il existe une relation entre les décisions des individus et le contexte dans lequel ils opèrent, de sorte que des facteurs tels que la perception de la richesse, la confiance institutionnelle et la victimisation sont décisifs. Cette recherche contribue ainsi à des études antérieures sur la prise des décisions économiques qui contredisent le modèle classique de la maximisation du profit.

Mots clés: comportement; l'honnêteté; tricherie; expérimentation.

Classification JEL: D01, D73, C93. 


\title{
El contexto de la trampa: análisis experimental de los determinantes de la honestidad en Buriticá, Colombia*
}

\author{
Adolfo Eslava $\mathbb{Q}^{\mathrm{a}}$ y Estefanía García-Luna $\mathbb{\circledR}^{\mathrm{b}}$ \\ -Introducción. -I. Marco teórico. -II. Metodología. -III. Resultados. -Conclusiones. \\ -Anexos. -Referencias.
}

doi: 10.17533/udea.le.n92a01

Primera versión recibida el 24 de agosto de 2018; versión final aceptada el 11 de junio de 2019

\section{Introducción}

La trampa, los actos deshonestos y los actos al margen de la ley se observan como una constante de la vida cotidiana en diferentes lugares y escenarios (Bucciol \& Piovesan, 2008). Más allá de las implicaciones de estas decisiones en la vida de los individuos que las toman, los actos deshonestos son altamente costosos para la sociedad (Mazar \& Ariely, 2006).

En particular, la minería aurífera en Colombia se ha caracterizado por contar con una tradición de explotación informal que configura escenarios al margen de la ley; sin embargo, a pesar de los problemas que se generan en los territorios, existe un capital social (atributos de comunidad) que permite a la comunidad hacerles frente (Eslava, 2014).

El municipio antioqueño de Buriticá ha sufrido cambios en sus dinámicas como consecuencia de la reciente explotación minera. A pesar de no poseer

* Artículo derivado de la investigación "Gobernanza de la Minería Aurífera en Buriticá. Comprender microfundamentos comunitarios" de la Universidad EAFIT y cofinanciado por la Fundación Proantioquia.

a Adolfo Eslava: profesor titular, Departamento de Gobierno y Ciencias Políticas, Universidad EAFIT, Colombia. Dirección electrónica: aeslava@eafit.edu.co https:/ / orcid.org/0000-0003-2213-0765

b Estefanía García-Luna: auxiliar de investigación, Departamento de Gobierno y Ciencias Políticas, Universidad EAFIT, Colombia. Dirección electrónica: egarcia5@eafit.edu.co https://orcid.org/0000-0002-2296-6071 
Eslava y García-Luna: El contexto de la trampa: análisis experimental de los determinantes...

cifras oficiales, se estima que la población flotante en el municipio se duplicó a causa de los mineros no legalizados que migraron en búsqueda de oportunidades (Tamayo, 2017).

La vocación económica del municipio, que ha sido principalmente agrícola, incluye ahora la minería, ejercida por informales, actores ilegales y por la empresa que posee la licencia de explotación: la multinacional canadiense Continental Gold (Silva, 2015). Lo anterior, sumado a las condiciones socioeconómicas de las cuales dan cuenta los bajos indicadores de desempeño municipal (DNP, 2016) y los altos índices de pobreza (70,3\% de la población con necesidades básicas insatisfechas -DANE, 2005-) exponen algunos problemas en los que el municipio se ve envuelto.

Frente a este contexto, la comunidad, como sujeto activo que establece instituciones y puede generar dinámicas productivas o destructivas (Ostrom, 2013), cuenta con atributos propios para afrontar los retos. No indagar por estos atributos y sus determinantes entorpece la aplicación de incentivos que promuevan comportamientos beneficiosos para la comunidad.

Más que pensar en las soluciones que se pueden tomar desde el gobierno, "es necesario reconocer y comprender la microinstitucionalidad que caracteriza la vida cotidiana de las personas que habitan los territorios mineros" (Eslava, 2014). Para responder a esta necesidad de estudio, se resalta la obligación que tiene la economía de estudiar y entender cualquier tipo de decisión humana (Sala i Martin, 2016).

Ser honestos, como acción opuesta a la trampa, se reconoce como un comportamiento que elimina altos costos sociales (Ibáñez \& Vásquez, 2014) y que, por ende, puede generar consecuencias favorables para los habitantes del municipio. Por esta razón, se pretende aquí estudiar el comportamiento de los buritiqueños, a través de un experimento económico que analiza la honestidad y su relación con hechos victimizantes y características prosociales.

A continuación, se realiza una revisión de los fundamentos teóricos de la economía del comportamiento, dentro de los que se reconoce el papel de la honestidad; se presenta también la metodología usada para su medición en el municipio de Buriticá y se plantea una discusión sobre los resultados del experimento. 


\section{Marco teórico}

En la economía del comportamiento se reconoce la honestidad como un factor importante en diferentes escenarios: en el económico, es relevante la forma en la que los agentes reportan sus ingresos al pagar o evadir impuestos se hace relevante para las autoridades; en el político, los votantes le dan importancia a la probabilidad de que alguien que llegue al poder se vuelva corrupto; y en general, en cualquier escenario, la capacidad de evaluar con precisión la probabilidad de mentir de los demás es crucial para tomar buenas decisiones (Fischbacher \& Heusi, 2008).

La importancia del comportamiento deshonesto se ha abordado en diferentes contextos: académicos, de relaciones amorosas, económicos, entre otros (Azar, Yosef \& Bar-Eli, 2013). En economía, la deshonestidad se ha abordado ampliamente a nivel institucional (corrupción), pero, recientemente ha tomado protagonismo el análisis de este tipo de cualidades y acciones a nivel individual (Pascual-Ezama et al., 2015).

Con la introducción de un hombre con racionalidad limitada en la economía conductual, se confirma la importancia de un análisis que evalúe los determinantes del comportamiento del hombre y busque promover aquellos que lleven a un bienestar social en el largo plazo. De allí que la forma de abordar este objetivo sea a través de las políticas públicas, pues estas tienen entre sus finalidades facilitar el desarrollo de instituciones a través de intervenciones diseñadas y teniendo en cuenta las capacidades existentes en la comunidad, o ayudar a construir esa capacidad antes de intervenir (Ostrom, 2013).

Los actos deshonestos producen recompensas en el corto plazo a quienes los cometen (Blanco \& Cárdenas, 2015), y a pesar de las leyes existentes para desincentivar estos comportamientos, la teoría económica define que las personas simplemente mienten cuando tienen la oportunidad de hacerlo, aunque solo hasta cierto punto; esto es, un nivel que varía para cada individuo (Ibáñez \& Vásquez, 2014).

En el modelo estándar de la economía racional se forma la creencia de que las personas llevan a cabo actos deshonestos de forma consciente y deliberada, 
Eslava y García-Luna: El contexto de la trampa: análisis experimental de los determinantes...

haciendo un balance entre los beneficios externos que se pueden obtener y los costos del acto deshonesto (Mazar, Amin \& Ariely, 2008). Desde la perspectiva del Homo Economicus se asume que el individuo sabe lo que quiere y, de esta forma, es capaz de comportarse de modo que sus decisiones le generen mayores utilidades (Mazar \& Ariely, 2006). Esto implicaría que las decisiones relativas a la honestidad son como cualquier otra decisión de los individuos. Es más, como afirman Mazar y Ariely (2006), desde esta perspectiva, los esfuerzos para limitar acciones como la trampa y el fraude se han hecho desde la ley, pues se asume que la única forma de progresar en ello es aumentar los costos y restringir los beneficios de los actos deshonestos.

En contraste, la psicología asume que las personas se interesan también por sus recompensas internas, ya que quieren mantener un buen concepto de sí mismas. Como lo exponen Nina Mazar y Dan Ariely (2006), esta visión se apoya en otras ciencias como la sociología, la antropología, y la economía experimental y del comportamiento, en las cuales se asegura que este mecanismo de recompensa interno hace que la forma en que las personas se comportan muestre reciprocidad y altruismo cuando se enfrentan a dilemas sociales.

Se crea entonces una dicotomía entre las ganancias de la trampa, por un lado, y la conservación de un concepto positivo de sí mismo, por otro lado. Adicionalmente, se debe agregar otra variable en la ecuación de los incentivos para hacer o dejar de hacer trampa: el reconocimiento social.

Las personas, en la mayoría de las sociedades, valoran la honestidad (Mazar et al., 2008). Así, cuando existen espectadores que pueden detectar y castigar el comportamiento inmoral, este se puede deteriorar, y dada la preocupación por la forma en que los demás nos perciben, la forma de actuar puede cambiar para mantener una imagen social (Azar et al., 2013).

Cabe aquí preguntar por qué comportarse de acuerdo con esa regla social. Una regla separa los comportamientos posibles en dos grupos: los que están conformes a ella o contrarios a ella. Con base en esto, y en lo descrito en los párrafos anteriores, existen tres tipos de regulación que contribuyen al análisis cualitativo de los determinantes de la honestidad: legal, moral y cultural (Mockus, 1999). Esta trilogía regulativa hace que las personas se comporten 
como anfibios culturales que se mueven discrecionalmente en los ambientes institucional, personal y social.

Las ideas anteriores exponen entonces cómo las preferencias a la hora de tomar decisiones económicas no son exógenas. Contrario a lo que plantea el modelo clásico de la economía, estas preferencias se forman por las interacciones sociales y económicas (Henrich et al., 2001). Esta es la razón por la cual en el ejercicio experimental aquí propuesto se evalúan la prosocialidad - entendida como aquellas acciones que, si se practican con frecuencia, mejoran el bienestar promedio de una comunidad (Bowles \& Gintis, 1997) - y la victimización — que hace referencia a qué tanto las personas han sido víctimas de delitos contra la integridad, la propiedad o la vida (Velásquez et al., 2012) —. Para esto se tiene en cuenta el trabajo elaborado en Oro como Fortuna (2014), donde estas variables resultan determinantes al evaluar las preferencias de los individuos.

De lo anterior se desprende que, algunas recomendaciones de política pública dependan del análisis de las causas de la trampa para ser acertadas y disminuir los comportamientos deshonestos. Este análisis tiene consecuencias para los fenómenos económicos, en casos como el diseño óptimo de instancias y contratos, la asignación de derechos de propiedad y las condiciones para una acción colectiva exitosa (Henrich et al., 2001).

Sea que la causa principal de la deshonestidad se dé por los bajos costos externos y los altos beneficios, o por los vacíos en las normas sociales que resulten en un mecanismo de recompensa interna débil (Mazar et al., 2008), es necesario entender estos rasgos comunitarios para establecer decisiones públicas que puedan abarcar la acción colectiva. Además, "comprender las motivaciones de una persona - altruismo, reciprocidad, aversión a la desigualdad y el interés personal- es esencial para poder predecir cómo se comportarán al ser empleados, miembros de una familia, guardianes del medio ambiente y ciudadanos" (CORE, 2017).

Para entender dicho comportamiento económico e indagar por las preferencias de los individuos, los experimentos conductuales se han convertido en protagonistas, ya que las decisiones que se toman en ellos tienen instrucciones, reglas comunes a todos los participantes, incentivos y 
Eslava y García-Luna: El contexto de la trampa: análisis experimental de los determinantes...

consecuencias, además pueden ser replicados en diferentes situaciones: "Esto significa que cuando las personas se comportan de manera diferente en el experimento, es probable que se deba a diferencias en sus preferencias, no a la situación que cada persona enfrenta" (CORE, 2017, 4.8).

Es evidente entonces la pertinencia de los experimentos económicos para estudiar la honestidad, entendida como atributo opuesto a la tendencia a la trampa, la cual permite reducir incertidumbre y costos de transacción en las interacciones cotidianas, para ello es necesaria la evaluación de sus determinantes como insumo a la literatura estudiada.

\section{Metodología}

Para el estudio de los determinantes de la honestidad en Buriticá, se realizó un experimento de laboratorio en campo con el objetivo de recolectar los datos que permiten la medición de la probabilidad que tienen los participantes de hacer trampa o comportarse de forma honesta (véase Anexo 1).

Es pertinente resaltar que el experimento brinda ventajas como el hecho de que se pueden capturar preferencias individuales basadas en el comportamiento observado y no en la percepción, dado que existen incentivos monetarios (Eslava, 2014).

Este experimento - que consiste en reportar de forma privada el resultado que se obtiene al lanzar un dado, a cambio de una recompensa que será mayor mientras más alto sea el valor declarado- es réplica de los experimentos que se han llevado a cabo en los últimos años para medir la honestidad, los cuales tienen como base la economía experimental y las ciencias del comportamiento (Blanco \& Cárdenas, 2015; Eslava, 2014; Fischbacher \& Heusi, 2008; Ibáñez \& Vásquez, 2014; Pascual-Ezama et al., 2015). Además, en esta investigación se incluye una encuesta posjuego de la cual se desprende la información con la que se elabora el modelo econométrico (véase Anexo 2).

A partir de los datos recopilados, se realiza un análisis de la distribución de los resultados reportados al lanzar el dado, para así compararla con la distribución uniforme que se debería obtener bajo el supuesto de honestidad. 
De esta forma se evalúa la existencia de una tendencia a la trampa dentro de los participantes (Ibañez \& Vásquez, 2014; Blanco \& Cárdenas, 2015), pues se espera que en un escenario como este, en el que no hay sanciones, los participantes maximicen sus ganancias y reporten el valor que más utilidad monetaria les genere sin importar el resultado del lanzamiento.

Con la frecuencia de los lanzamientos y los datos recogidos en la encuesta posjuego, se plantea un modelo donde la variable dependiente corresponde a la probabilidad de reportar cada valor del dado. Así, se determina la probabilidad de reportar cada valor a partir de las variables del contexto y los atributos de los participantes (Cohn, Maréchal \& Noll, 2015; Ibáñez \& Vásquez, 2014).

A diferencia de los autores mencionados, que usan un modelo de regresión lineal (mínimos cuadrados ordinarios) para el análisis de la variable dependiente, se propone un modelo probit ordenado, porque este responde a la necesidad de plantear las probabilidades frente a seis categorías específicas, a saber, los seis valores que se pueden reportar sumando los puntos de las caras de los dados. También se usa este método para minimizar el riesgo del uso de modelos de probabilidad lineal que engloban una naturaleza heterocedástica, que pueden arrojar resultados de probabilidades que no se encuentran acotados entre 0 y 1 , y que, además, consideran efectos marginales constantes (Wooldridge, 2009).

El modelo a estimar se representa de la siguiente forma:

$$
\begin{aligned}
\operatorname{Pr}(\boldsymbol{T})=\boldsymbol{\alpha}+\boldsymbol{\beta} \text { Victimizacion }+\boldsymbol{\gamma} \text { Prosocialidad } & \\
& +\boldsymbol{\delta} \text { Socioeconomicas }+\varepsilon
\end{aligned}
$$

Así, a través del probit ordenado, se determinará cómo la victimización y los atributos prosociales influyen en la probabilidad de reportar un valor específico del dado, $\operatorname{Pr}(\boldsymbol{T})$.

La variable victimización (Victimizacion) es tratada como una dummie para aquellos hechos más representativos, a saber: hurto y desaparición de un familiar. 
Eslava y García-Luna: El contexto de la trampa: análisis experimental de los determinantes...

Los atributos prosociales (Prosocialidad) se identifican a través de la confianza que los participantes dicen tener en la Policía Nacional, medida en una escala de 1 a 4 (donde 1 es "nada", 2 es "poco", 3 es "algo" y 4 es "mucho"), y a través de la pertenencia a un grupo comunitario debido a la ausencia del Estado en el municipio, tratada como una dummie.

Se incluye también como regresor el nivel de pobreza (Socioeconomicas) estudiado a través de la percepción propia de pobreza que se marca en una escala de 1 a 10, donde 1 significa el nivel más alto de pobreza y 10 el nivel más alto de riqueza.

\section{Resultados}

La metodología descrita fue aplicada en cinco sesiones, cada una de las cuales contó con la participación de 17 personas en promedio, representantes de los actores relevantes en los procesos sociales del municipio.

A continuación, se da cuenta de las características sociodemográficas de los participantes en cada una de las sesiones (véase Tabla 1).

Frente a los resultados reportados por los participantes del experimento, se encuentra una distribución con una mayor frecuencia de los valores 4, 5 y 6, como se muestra a en la Figura 1.

Figura 1. Frecuencia de valores reportados en el experimento

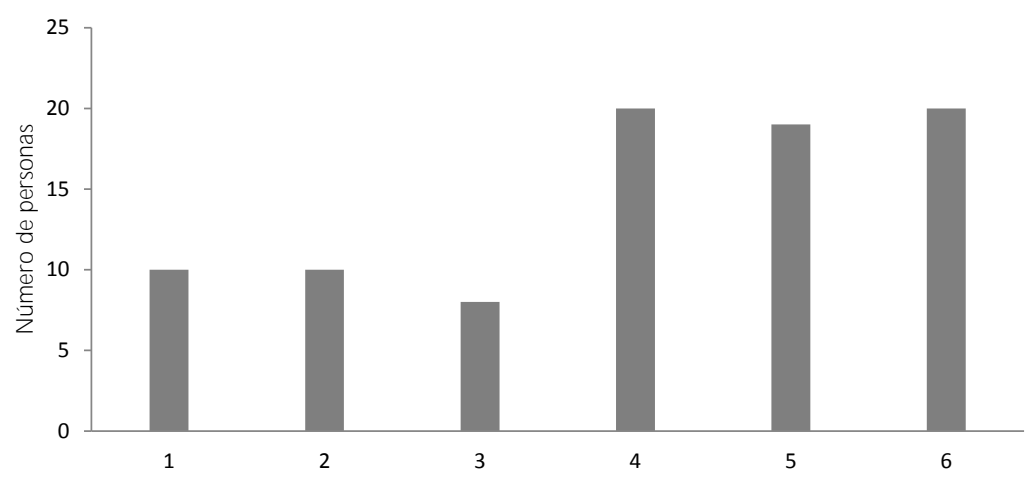

Fuente: elaboración propia. 
Tabla 1. Características sociodemográficas

\begin{tabular}{lcccccc}
\hline Sesión & 1 & 2 & 3 & 4 & 5 & Total \\
\hline Participantes & 18 & 19 & 18 & 16 & 16 & $\mathbf{8 7}$
\end{tabular}

\section{Edad}

14-30

$\begin{array}{llllll}39 \% & 26 \% & 28 \% & 13 \% & 25 \% & \mathbf{3 8} \% \\ 28 \% & 32 \% & 28 \% & 63 \% & 56 \% & \mathbf{4 7 \%} \\ 33 \% & 42 \% & 44 \% & 25 \% & 19 \% & \mathbf{1 5 \%}\end{array}$

51-77

\section{Género}

Femenino

$\begin{array}{llllll}67 \% & 42 \% & 67 \% & 50 \% & 69 \% & \mathbf{5 9} \%\end{array}$

Masculino

$\begin{array}{llllll}33 \% & 58 \% & 33 \% & 50 \% & 31 \% & \mathbf{4 1} \%\end{array}$

\section{Escolaridad}

Inferior a primaria

$\begin{array}{llllll}11 \% & 0 \% & 39 \% & 0 \% & 0 \% & \mathbf{1 0} \%\end{array}$

Primaria completa

$\begin{array}{llllll}33 \% & 11 \% & 33 \% & 13 \% & 0 \% & \mathbf{1 8} \%\end{array}$

Secundaria completa

$17 \% \quad 47 \% \quad 0 \% \quad 0 \% \quad 0 \% \quad \mathbf{1 4} \%$

Técnico/Tecnólogo

$\begin{array}{llllll}28 \% & 32 \% & 17 \% & 31 \% & 6 \% & \mathbf{2 3} \%\end{array}$

Profesional

$\begin{array}{llllll}11 \% & 11 \% & 6 \% & 44 \% & 44 \% & \mathbf{2 2} \%\end{array}$

Postgrado

$\begin{array}{llllll}0 \% & 0 \% & 6 \% & 13 \% & 50 \% & \mathbf{1 3} \%\end{array}$

\section{Distribución de ingresos}

Ingresos suficientes sin dificultades $\begin{array}{rrrrrr}5 \% & 11 \% & 17 \% & 13 \% & 37 \% & \mathbf{1 6 \%} \\ 50 \% & 47 \% & 17 \% & 50 \% & 63 \% & \mathbf{4 5 \%} \\ 28 \% & 42 \% & 33 \% & 31 \% & 0 \% & \mathbf{2 8 \%} \\ 17 \% & 0 \% & 33 \% & 6 \% & 0 \% & \mathbf{1 1} \%\end{array}$ Ingreso no suficiente y con grandes dificultades $\quad 17 \% \quad \begin{array}{lllllll}0 & 33 \% & 6 \% & 0 \% & \mathbf{1 1} \%\end{array}$ Autopercepción de pobreza

$\begin{array}{llllll}4 & 6 & 3 & 5 & 7 & 5\end{array}$

Fuente: elaboración propia.

Si bien los datos fueron recolectados en sesiones diferentes, el análisis econométrico se elabora sobre el agregado de los resultados que se 
Eslava y García-Luna: El contexto de la trampa: análisis experimental de los determinantes...

muestra en la Figura 1. De esta forma, al evaluar las probabilidades de reportar cada número, a través de una prueba de Kolmogorov-Smirnov, con un valor significativo al $1 \%$, se encuentra que la distribución de la probabilidad predicha de que los jugadores reporten cada número no corresponde a la distribución que se obtendría bajo el supuesto de honestidad (véase Figura 2).

Figura 2. Probabilidades predichas vs Probabilidad uniforme

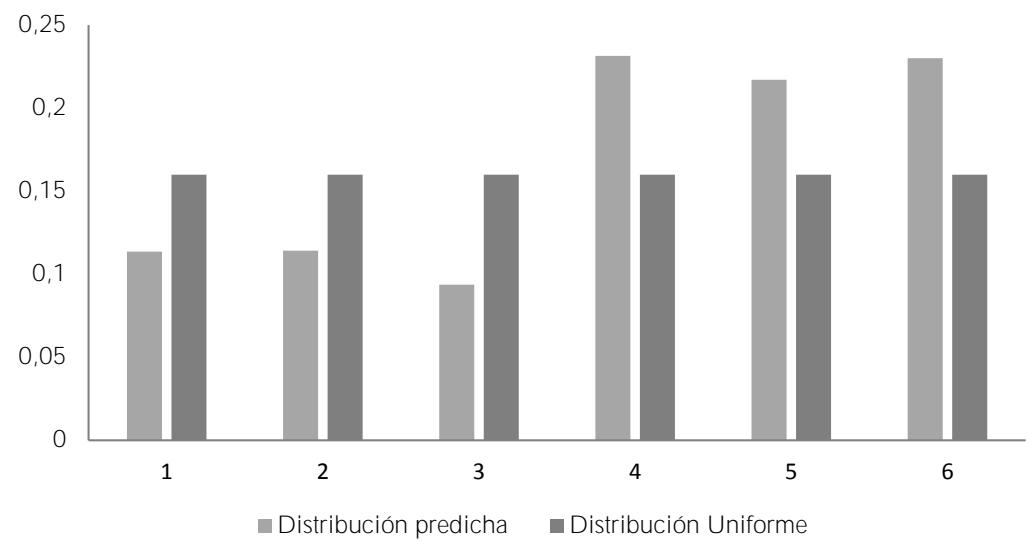

Fuente: elaboración propia.

A pesar de la distribución encontrada (en la que se evidencia una maximización en las ganancias de los jugadores, obtenidas al reportarse de forma más alta los valores 4, 5 y 6), la existencia de la frecuencia de los valores 1,2 y 3 se puede interpretar como un hecho que da cuenta de un nivel bajo de trampa, si se considera que en este tipo de experimentos no existe una sanción frente a los valores reportados y que cada participante cuenta con privacidad y anonimato (Ariely, 2012; Eslava, 2014).

Se evidencia también un balance entre las perspectivas económicas y las comportamentales mencionadas, lo que contradice el supuesto racional de la maximización de las ganancias al reportar el valor más alto (6), pues los valores 4 y 5 contaron con una alta frecuencia. De esta manera se hace "un poco" de trampa y se obtiene un beneficio de ello, pero se mantiene una imagen positiva de sí mismo (Ariely, 2012). 
A continuación, se presentan los resultados obtenidos a través del probit ordenado con los efectos marginales calculados para cada número reportado, esto es, el efecto sobre la probabilidad de observar las variables ordenadas (véase Tabla 2).

Tabla 2. Resultados del modelo probit ordenado

\begin{tabular}{lcccccccc}
\hline \multicolumn{7}{c}{ Variable dependiente: número reportado } \\
\hline & \multirow{7}{*}{$\begin{array}{c}\text { Coeficiente } \\
\text { estimado }\end{array}$} & \multicolumn{7}{c}{ Efectos marginales } \\
\cline { 3 - 8 } & $-0,68^{*}$ & 0,15 & $0,07^{*}$ & $0,02^{* *}$ & $-0,01$ & $-0,09$ & $-0,15^{* *}$ \\
\hline \multirow{2}{*}{ Desaparición de un familiar } & $0,37)$ & $(0,11)$ & $(0,04)$ & $(0,01)$ & $(0,03)$ & $(0,06)$ & $(0,06)$ \\
\hline \multirow{2}{*}{ Hurto } & $0,51^{*}$ & $-0,06^{*}$ & $-0,05^{*}$ & $-0,03$ & $-0,03$ & $0,03^{* *}$ & 0,16 \\
& $(0,29)$ & $(0,03)$ & $(0,03)$ & $(0,02)$ & $(0,03)$ & $(0,02)$ & $(0,10)$ \\
\hline \multirow{2}{*}{ Confianza en la } & $-0,23^{*}$ & $0,03^{*}$ & $0,02^{*}$ & 0,01 & 0,01 & $-0,25$ & $-0,06^{*}$ \\
Policía Nacional & $(0,12)$ & $(0,02)$ & $(0,01)$ & $0,01)$ & $(0,01)$ & $(0,01)$ & $(0,03)$ \\
\hline \multirow{2}{*}{ Estado no sirve } & $-0,63^{*}$ & 0,13 & $0,07^{*}$ & $0,03^{* *}$ & $-0,01$ & $-0,08$ & $-0,14^{* *}$ \\
& $(0,34)$ & $(0,09)$ & $(0,04)$ & $(0,01)$ & $(0,02)$ & $(0,05)$ & $(0,06)$ \\
\hline \multirow{2}{*}{ Escala de pobreza } & $-0,15^{* * *}$ & $0,02^{* *}$ & $0,01^{* *}$ & $0,01^{* *}$ & 0,01 & $-0,01^{* *}$ & $-0,04^{* * *}$ \\
& $(0,05)$ & $(0,01)$ & $(0,01)$ & $(0,01)$ & $(0,01)$ & $(0,01)$ & $(0,01)$ \\
\hline Pseudo $R^{2}$ & 0,05 & & & & & & \\
\hline
\end{tabular}

Nota: ${ }^{* *}$ Significativo al $1 \%,{ }^{*}$ Significativo al $5 \%,{ }^{*}$ Significativo al $10 \%$.

Fuente: elaboración propia.

De esta forma, se observa que ser víctima de hurto se relaciona de forma positiva con reportar valores más altos en el juego ( 5 y 6 ), pues la probabilidad de reportar un valor mayor aumenta 0,03 y 0,16 puntos porcentuales respectivamente. Por otro lado, sufrir la desaparición de un familiar disminuye la probabilidad de reportar estos mismos valores en 0,09 y 0,15 puntos porcentuales. 
Eslava y García-Luna: El contexto de la trampa: análisis experimental de los determinantes...

Esto da cuenta de la importancia de marcar diferencias entre los tipos de victimización, pues se muestra cómo la relación con la variable dependiente no es igual para ambos casos. Una explicación a esto la dan autores como Russo y Roccato (2010) o Bar-Tal (2007), quienes resaltan la diferencia de los efectos de la victimización indirecta y directa, siendo esta última la que más influye en la capacidad de reacción y el comportamiento de quienes la padecen.

Continuando con los datos obtenidos, frente a las variables prosociales, se tiene que mientras se declare una mayor confianza en la Policía Nacional, la probabilidad de reportar valores altos, como el 5, disminuye en 25 puntos porcentuales. Igualmente ocurre con el hecho de ser parte de un grupo comunitario debido a la poca presencia del Estado en el municipio, pues también disminuye la probabilidad de reportar los valores altos, 4, 5 y 6 , en un $1 \%, 8 \%$ y $14 \%$, respectivamente.

Este último grupo de variables da cuenta de la importancia de la fortaleza institucional dentro del municipio, aún más cuando la honestidad se considera como un factor particularmente importante en un ambiente con una baja institucionalidad (Hugh-Jones, 2015).

Por último, con una alta significancia, se encuentra que el valor de la escala de pobreza, esto es, la autopercepción de las personas de su condición de pobreza o riqueza, impacta en el valor reportado de forma negativa. Las personas que consideran tienen mayor riqueza, tienen una probabilidad de 0,01 y 0,04 puntos porcentuales menor de reportar los valores más altos.

\section{Conclusiones}

Los resultados encontrados en este trabajo son ejemplo de la relación que existe entre el contexto en el que se desenvuelven las personas y la toma de decisiones de los individuos. Así como lo explican las diferentes ciencias del comportamiento, no solo se actúa pensando en la maximización de los beneficios propios, sino que en las decisiones y acciones individuales también se consideran el contexto y a los demás. 
La honestidad, desde los hallazgos presentados, se determina en gran medida por la confianza en las instituciones. Bajo el reconocimiento de este atributo de comunidad como insumo para mejorar las dinámicas en el municipio de Buriticá, se señala la pertinencia del fortalecimiento de dicha confianza, dado que cuando las instituciones se perciben como débiles, es menos probable que las personas se adapten a ellas o las sigan (Vetter, Houser \& Winter, 2012).

Se resalta, además, que este artículo contribuye a la literatura estudiada dado el contexto concreto donde se realiza el experimento, pues es un escenario donde predominan fenómenos de desafección política, y se condenan las relaciones entre habitantes a que estén permeadas por la trampa; sin embrago, se descubre que, aún en este contexto, puede haber honestidad. La contribución entonces no es teórica, se trata de la aplicación de herramientas a un contexto local que requiere ser comprendido desde las diferentes posibilidades que ofrece la literatura.

Si bien los resultados se corresponden con los estudios analizados y esto favorece la robustez de los hallazgos, se deja abierta la discusión sobre el estudio, bajo el modelo experimental, de los determinantes de la honestidad en la economía. Es pertinente continuar indagando por la relación que presenta la tendencia a la honestidad con la victimización, la confianza en las instituciones y las características socioeconómicas de los individuos, esto, para avanzar en la comprensión de la incidencia del contexto en las decisiones individuales que pueden favorecer el bienestar social.

\section{Anexo 1. Protocolo del ejercicio experimental}

Por medio del presente texto se busca brindar a los miembros del proyecto las herramientas conceptuales y metodológicas necesarias para llevar a cabo de forma eficiente el experimento económico. A partir del cumplimiento de lo estipulado aquí, se dotará de robustez a la recolección de datos en campo y además se posibilitará un desarrollo consistente y ordenado de las jornadas.

Durante las cinco sesiones experimentales se convocan máximo 30 personas de diferentes grupos representativos para la toma de decisiones 
Eslava y García-Luna: El contexto de la trampa: análisis experimental de los determinantes...

en el municipio, estos son: funcionarios públicos, mineros en proceso de formalización, personas de veredas mineras, trabajadores de la empresa minera y personas de la zona urbana del municipio.

En el momento de la llegada de los participantes a cada sesión, estos se dirigirán a la mesa de registro donde se tomarán los siguientes datos: nombre, documento de identidad y teléfono.

Después de este primer registro, otro miembro del equipo se encontrará diligenciando el formato prejuego en el que se preguntan los siguientes datos: edad, último nivel educativo cursado y sexo. En este punto se les entregará un ficho con el número de jugador asignado y el lapicero con el cual llenarán los formatos.

Luego de 20 minutos de espera y después de haber diligenciado los anteriores datos, se procede a comenzar la actividad.

Se inicia mencionando que este tipo de ejercicios no tienen una forma correcta de resolverse. Son juegos de toma de decisiones, en los cuales es fundamental que los participantes se sientan libres de decidir. Se les explica que, según la decisión que tomen durante la jornada, podrán recibir un monto de dinero en efectivo, de allí la importancia de que presten atención y piensen muy bien lo que van a decidir, ya que de dichas decisiones dependerán sus ganancias.

Se aclara también que la información que proporcionen para el estudio será confidencial y su uso está destinado a fines académicos. Tanto su nombre como número de identificación serán omitidos en cualquier informe o reporte que surja del estudio. Para ello, se solicita que no muestren el número de jugador asignado a las demás personas y que mantenga las hojas que se les dan boca abajo hasta que se indique lo contrario.

Los participantes deben saber que en cualquier momento pueden retirarse del ejercicio, sin embargo, el dinero solo pagará al final de la sesión y dependerá de completar tanto el ejercicio como la encuesta posjuego.

Ante cualquier duda que se presente durante el ejercicio, se les explicará las veces que lo requieran. Las preguntas que tengan una relación con los ejercicios serán expresadas y resueltas en voz alta, esto con el fin de que 
todos los participantes tengan la misma información a la hora de tomar sus decisiones.

Después de que se brinda la información anterior, y para asegurar que los jugadores quieren hacer parte del estudio, se les entrega un consentimiento informado. Esta hoja cuenta con la firma del coordinador de los ejercicios; esta firma garantiza la comodidad del participante y la seguridad de que la información que brindamos es cierta y será manejada con la discreción que ella requiere. Luego, se recoge el consentimiento y se da inicio al experimento.

A continuación, cada jugador recibe un vaso, un dado de seis caras y una hoja. El jugador debe lanzar el dado dentro del vaso una única vez. Después de lanzarlo, deberán observar por medio del agujero localizado en la tapa del vaso el valor obtenido. Únicamente el jugador verá el resultado de su lanzamiento, el cual deberá escribir en la hoja que se le entrega.

El pago de este ejercicio resulta del valor que arroje el dado multiplicado por $\$ 3.000$. Así, por ejemplo, si el dado arroja el número 1, el participante se llevaría $1 * \$ 3.000=\$ 3.000$, y si el dado arroja el número 6, usted se llevaría $6 * \$ 3.000=\$ 18.000$.

Se debe recordar nuevamente que el resultado del lanzamiento del dado es secreto y que solo lo verá el jugador que lo lanza; una vez realice el lanzamiento, debe reportar dicho resultado en la hoja entregada.

Los materiales serán recogidos y se les entregará la encuesta posjuego para que sea diligenciada. Mientras tanto, un miembro del equipo calcula las ganancias secretas que serán entregadas a cada jugador en un sobre cerrado cuando finalicen la encuesta.

\section{Anexo 2. Encuesta posjuego}

Número de sesión

Número de jugador

1. ¿Hace cuántos años vive en este municipio? 
Eslava y García-Luna: El contexto de la trampa: análisis experimental de los determinantes...

2. Imagínese una escalera de 10 peldaños, en que en el "1" se ubican los hogares más pobres de su municipio y en el "10" se ubican los hogares con mayor riqueza de su municipio, ¿dónde se ubicaría su hogar?

Muy pobre

Muy rico

\begin{tabular}{|l|l|l|l|l|l|l|l|l|l|}
\hline 1 & 2 & 3 & 4 & 5 & 6 & 7 & 8 & 9 & 10 \\
\hline
\end{tabular}

3. Su salario, y el ingreso total de su familia, cle permite cubrir todas sus necesidades de forma satisfactoria? ¿Cómo define esta situación? Seleccione una de las siguientes opciones:

__ Es más que suficiente y le alcanza para ahorrar

__ Es suficiente y no tiene grandes dificultades

_ No es suficiente y tiene dificultades

__ No es suficiente y tiene grandes dificultades

4. ¿Ha sido víctima de algunos de los siguientes delitos?

_ Hurto

_ Desplazamiento forzado

_ Homicidio de un familiar

__ Desaparición forzada de un familiar

__ Agresión física

__ Violencia sexual

_ Secuestro

_ _ Daño a su propiedad

__ Extorsión

_ Tortura

_ Ninguno

Otro

5. Después de leer todas las opciones, marque las tres problemáticas que considera son prioridades para el municipio de Buriticá.

Contaminación ambiental 
_ Corrupción

_ Desconfianza

_ Desempleo

_ Desigualdad

__ Inequidad de género

__ Inseguridad

_ Minería ilegal

__ Poca participación ciudadana

_ Poca presencia del Estado

Presencia de forasteros

6. ¿Participa en alguno de los siguientes grupos u organizaciones?

_ De caridad (no como beneficiario)

_ Organización Comunitaria o de barrio/vereda

_ Religiosa

__ Instancias de participación del Estado

_ Organización étnica

_ Cultural o deportiva

__ Asociaciones de padres de familia

__ Sindicato, cooperativa de trabajo o agremiación de productores

_ Movimiento o partido político

_ Ninguno

Otro

7. Entre las siguientes tres opciones, ¿cuál considera es la principal razón que lo motiva a participar en el grupo u organización?

_ Como ciudadano es un deber participar, es interesante y genera satisfacción

__ El Estado no ha servido para solucionar problemas, por lo tanto, es necesario participar

Para recibir beneficios personales o reconocimiento social 
Eslava y García-Luna: El contexto de la trampa: análisis experimental de los determinantes...

8. En términos generales, ¿diría usted que se puede confiar en la mayoría de las personas o que es necesario ser muy cuidadoso al tratar con la gente?

Se puede confiar en la mayoría de las personas.

Es necesario ser cuidadoso al tratar con la gente.

9. ¿Cuánto confía en los siguientes grupos?

\begin{tabular}{|l|l|l|l|l|}
\hline & Mucho & Algo & Poco & Nada \\
\hline Su familia & & & & \\
\hline Sus vecinos & & & & \\
\hline Su comunidad & & & & \\
\hline Líderes sociales & & & & \\
\hline Grupos ilegales & & & & \\
\hline Gente que conoce por primera vez & & & & \\
\hline
\end{tabular}

10. Si en un juego de moneda de "cara y sello" se ganara $\$ 100.000$ y se le propusiera seguir jugando qué haría:

Se queda con los $\$ 100.000$ y no juega más

Arriesga los $\$ 100.000$, buscando ganar otros $\$ 100.000$, pero con el riesgo de perderlo todo si no acierta en la nueva ronda.

11. ¿Cuánto confía en las siguientes instituciones?

\begin{tabular}{|l|l|l|l|l|l|}
\hline & Mucho & Algo & Poco & Nada & NS/NR \\
\hline Ejército & & & & & \\
\hline Sistema Judicial & & & & & \\
\hline Gobierno Nacional & & & & & \\
\hline Concejo Municipal & & & & & \\
\hline Gobernación & & & & & \\
\hline Policía Nacional & & & & & \\
\hline Iglesia Católica & & & & & \\
\hline Presidente & & & & & \\
\hline Alcaldía & & & & & \\
\hline Universidades & & & & & \\
\hline Fiscalía General & & & & & \\
\hline Organizaciones Sociales & & & & & \\
\hline Juntas de acción comunal & & & & & \\
\hline EPM & & & & & \\
\hline Continental Gold & & & & & \\
\hline
\end{tabular}


12. ¿Entendió las instrucciones de las actividades? Sí

No

13. ¿Qué motivó las decisiones en los juegos?

_ Beneficio propio

Beneficio del grupo

_ Confianza en los demás

_ Incertidumbre

Emociones

\section{Referencias}

Ariely, D. (2012). The Honest Truth about Dishonesty. New York: Harper Collins.

Azar, O. H., Yosef, S. \& Bar-Eli, M. (2013). Do costumers return excessive change in a restaurant? A field experiment on dishonesty. Journal of Economic Behavior \& Organization, 93. Recuperado de: http://houdek petr.cz/!data/public_html/papers/Azar\%20et\%20al\%202013.pdf

Bar-Tal, D. (2007). Sociopsychological Foundations of Intractable Conflicts. American Behavioral Scientist, 50(11). Recuperado de: https://www.rese archgate.net/publication/258095712_Intractable_conflicts_Socio-psy chological_foundations_and_dynamics

Blanco, M. \& Cárdenas, J. C. (2015). Honesty after a labor relationship (Serie documentos de trabajo No.182). Recuperado de la Universidad del Rosario: http://repository.urosario.edu.co/bitstream/handle/10336/ 11303/dt182.pdf? sequence $=3$ \&isAllowed $=y$

Bucciol, A. \& Piovesan, M. (2008). Luck or Cheating? A Field Experiment on Honesty with Children. Journal of Economic Psychology, 32(1), 73-78. Recuperado de: https://www.sciencedirect.com/science/article/abs/pii/ S0167487010001352

Bowles, S. \& Gintis, H. (1998). The moral economy of communities: Structured populations and the evolution of pro-social norms. Evolution and Human Behavior, 19(1), 3-25. 
Eslava y García-Luna: El contexto de la trampa: análisis experimental de los determinantes...

Cohn, A., Maréchal, M. A. \& Noll, T. (2015). Bad boys: How criminal identity salience affects rule violation (Working paper No.132). Recuperado de Universidad de Zurich: http://www.econ.uzh.ch/static/wp/econwp 132.pdf

CORE (2017). A combination of self-interest, a regard for the wellbeing of others, and appropriate institutions can yield desirable social outcomes when people interact. Recuperado de: http://www.core-econ.org/the-economy/book /text/04.html

Departamento Nacional de Estadísticas (2005). Necesidades Básicas Insatisfechas - desagregada. Recuperado de: http://www.dane.gov.co/index.php/ estadisticas-por-tema/pobreza-y-condiciones-de-vida/necesidades-basi cas-insatisfechas-nbi

Departamento Nacional de Planeación (2016). Fichas territoriales: Buriticá. Recuperado de: https://terridata.dnp.gov.co/index-app.html\#/perfiles/0 5113

Eslava, A. (Ed.) (2014). Oro como fortuna. Instituciones, capital social y gobernanza de la minería aurifera colombiana. Medellin: Universidad EAFIT, Departamento Administrativo de Ciencia, Tecnología e Innovación (COLCIENCIAS).

Fischbacher, U. \& Heusi, F. (2008). Lies in disguise: an experimental study on cheating (Research Paper Series No. 40). Recuperado de Thurgau Institute of Economics and Department of Economics at the University of Konstanz: https://pdfs.semanticscholar.org/228f/b7abf00354659cd5 1075bc6795ad67f64dfa.pdf

Henrich, J., Boyd, R., Bowles, S., Camerer, C., Fehr, E., Gintis, H. \& Mcelreath, R. (2001). In Search of Homo Economicus: Behavioral Experiments in 15 Small-Scale Societies. The American Economic Review, 91(2), 73-78. Recuperado de: http://pricetheory.uchicago.edu/levitt/Pa pers/HeinrichBoyd2001.pdf 
Hugh-Jones, D. (2016). Honesty and beliefs about honesty in 15 countries. Journal of Economic Behavior \& Organization, 127, 99-114. Recuperado de: https://www.sciencedirect.com/science/article/pii/S01672681163 0052X

Ibáńez, M. \& Vásquez, J. (2014). How alternative development spills-over into more honest communities: An experimental approach in Colombia. En Beyond punishment: Achieving sustainable compliance with the law. The case of coca-reducing policies in Colombia (Tesis doctoral, Georg-AugustUniversität Göttingen). Recuperado de: https://bit.ly/30uAZkA

Mazar, N. \& Ariely, D. (2006). Dishonesty in everyday life and its policy implications. Journal of Public Policy \& Marketing, 25(1), 117-126. Recuperado de: https://www-2.rotman.utoronto.ca/facbios/file/Ma zar_JPPM_2006.pdf

Mazar, N., Amir, O. \& Ariely, D. (2008). The dishonesty of honest people: a theory of self-concept maintenance. Journal of Marketing Research, $X L V(6)$, 633-644. Recuperado de: http://people.duke.edu/ -dandan/ webfiles/PapersPI/Dishonesty\%20of\%20Honest\%20People.pdf

Mockus, A. (1999). Armonizar ley, moral y cultura: Cultura ciudadana, prioridad de gobierno con resultados en prevención y control de violencia en Bogotá, 1995-1997. Recuperado de: https://publications.iadb.org/es /publicacion/15999/armonizar-ley-moral-y-cultura-cultura-ciudadana -prioridad-de-gobierno-con

Ostrom, E. (2013). Más allá de los mercados y los Estados: Gobernanza policéntrica de sistemas económicos complejos. Revista de Derecho Ambiental de la Universidad de Palermo, II(1), 5-72. Recuperado de: http://www.palermo.edu/derecho/pdf/publicaciones/DA_N2_01.pdf

Pascual-Ezama, D., Fosgaard, T. R., Cardenas, J. C., Kujal, P., Veszteg, R., Gil-Gómez, B., Gunia, B., Weichselbaumer, D., Hilken, K., Antinyan, A., Delnoij, J., Proestakis, A., Tira, M. D., Pratomo, Y., JaberLópez, T. \& Brañas-Garza, P. (2015). Context dependent cheating: Experimental evidence from 16 countries. Journal of Economic Behavior 
Eslava y García-Luna: El contexto de la trampa: análisis experimental de los determinantes...

\& Organization, 116, 379-386. Recuperado de: https://www.sciencedir ect.com/science/article/pii/S0167268115001304

Russo, S. \& Roccato, M. (2010). How long does victimization foster fear of crime? A longitudinal study. Journal of Community Psychology, 38(8), 960-974. Recuperado de: https://www.researchgate.net/publication/2 29810364_How_long_does_victimization_foster_fear_of_crime_A_l ongitudinal_study

Sala I Martin, X. (2016). Economía en colores. España: Penguim Random House.

Silva Jaramillo, S. (2015). Minería aurifera y construcción de normas informales: análisis institucional del caso de Buriticá, Antioquia en 2009-2014. Entendiendo a los "emprendedores del incumplimiento" y su imposición de arreglos institucionales deshonestos (Tesis de maestría, Universidad EAFIT, Medellín, Colombia).

Tamayo Ortiz, H. (2017, 31 de julio). Investigan si hay manos criminales en explosión de mina en Antioquia. El Tiempo. Recuperado de: http: //www.eltiempo.com/colombia/medellin/investigacion-tras-explosionde-mina-de-buritica-en-antioquia-114852

Mockus, A., Murraín, H. \& Villa, M. (Coords.) (2012). Antípodas de la violencia: Desafios de cultura ciudadana para la crisis de (in)seguridad en América Latina. Banco Interamericano de Desarrollo. Recuperado de: https://publications.iadb.org/es/antipodas-de-la-violencia-desafiosde-cultura-ciudadana-para-la-crisis-de-inseguridad-en-america

Vetter, S., Houser, D. \& Winter, J. (2012). Fairness and Cheating. European Economic Review, 56(8), 1645-1655.

Wooldridge, J. M. (2009). Introducción a la Econometría: un enfoque moderno. México D.F., Cengage Learning. 